\title{
Diffractive Physics with ATLAS
}

\author{
A. Sidoti* \\ Istituto Nazionale Fisica Nucleare - Sezione Roma "La Sapienza", \\ P.le Aldo Moro 2, Rome, 00148, Italy \\ *E-mail: antonio.sidoti@roma1.infn.it \\ on behalf of the ATLAS collaboration
}

\begin{abstract}
In this paper we review diffractive physics measurements in LHC Run1 $p p$ collisions performed by the ATLAS experiment. We show the total inelastic proton-proton cross section, the differential cross section as a function of rapidity gaps and finally the dijet production with a veto on additional central jet activity.
\end{abstract}

Keywords: LHC; QCD; Diffraction; Atlas;

\section{Introduction}

Almost $\sim 30 \%$ of the inelastic proton-proton collisions at LHC at $\sqrt{s}=7 \mathrm{TeV}$ are diffractive processes. From a theoretical point of view, diffraction occurs when no quantum numbers are exchanged between the interacting particles. One description is that diffraction happens via the exchange of a Pomeron defined in the Regge phenomenology. The Pomeron is responsible for the increase of the total cross section with the increase of the centre-of-mass energy. Experimentally, diffraction is identified by two signatures: detection of elastically diffused protons and large rapidity gaps. Large rapidity gaps occur when there is no colour exchange in the diffractive interactions, therefore radiation (and thus produced particles) between the interacting particles is suppressed. As a function of rapidity gap, non-elastic events are exponentially suppressed while diffractive ones are not. Therefore large samples of diffractive events can be obtained requesting large rapidity gaps. The other experimental method used at ATLAS ${ }^{1}$ is the request that intact protons (one or both) emerge from the collisions. In this paper we will review the measurements that the ATLAS experiment performed on diffractive processes produced by protonproton collisions in the LHC Run1 (2009-2013). The paper is organized as follows: first the total measurement of the proton proton cross section using asymmetric events is presented. Then rapidty gaps results are presented. Prospects for the next LHC runs are summarized.

\section{Measurement of inelastic proton-proton cross section}

The inelastic $p p$ cross section has been measured by ATLAS at $\sqrt{s}=7 \mathrm{TeV}$ using events collected in 2010 runs with a mean number of interactions $\sim 0.01$ and an 
integrated luminosity of $20.3 \mu \mathrm{b}^{-1}$. This measurement uses highly efficient scintillation counters to detect very low momentum particles from inelastic collisions. Because of their pseudorapidity acceptance ${ }^{\mathrm{a}}$, these Minimum Bias Trigger Scintillator (MBTS $)^{2}$ are sensitive to diffractive dissociation processes to masses of the diffractive system $M_{X} \geq 15.7 \mathrm{GeV}$, corresponding to $\xi=\frac{M_{X}^{2}}{s}>5 \times 10^{-6}$. The total cross section measured in this kinematic region is:

$$
\sigma_{\text {inel }}\left(\xi>5 \times 10^{-6}\right)=60.33 \pm 0.05(\text { stat }) \pm 0.5(\text { syst }) \pm 2.1(\text { lumi }) \mathrm{mb}
$$

To compare with previous measurements performed at different $\sqrt{s}$ and with theoretical models, the measurement has to be extrapolated to the full kinematics region $\xi>\frac{m_{p}^{2}}{s}$. The extrapolation at low $M_{X}$ mass is performed using MC based on phenomenological models. This contribution is estimated measuring the ratio of singlesided events with respect to the total inelastic events. Single-sided events have at least two hits above a threshold well above the noise on the MBTS only on one side of the ATLAS detector ${ }^{\mathrm{b}}$. This measured ratio is $R_{s s}=10.04 \pm 0.03$ (stat) ${ }_{-0.4}^{+0.1}$ (syst) . The fractional contribution of diffractive events $f_{d}=\frac{\sigma_{S D}+\sigma_{D D}+\sigma_{C D}}{\sigma_{\text {inel }}}$ is constrained by the $R_{s s}$ measurement. For each MC model considered (PHOJET ${ }^{3,4}$, PYTHIA6 or PYTHIA8,...) that differ in the dependencies of the diffractive cross section with $\xi$, different $f_{D}$ values for the measured $R_{s s}$ are predicted. PYTHIA6 and PYTHIA8 differ in the modeling of the hadronic final state. The validity of the MC description is assessed by examining the hit multiplicity in the MBTS detector in the inclusive and single-sided event samples. The MC simulation PYTHIA $8+$ DL with $\alpha(0)=1.085$ and $\alpha^{\prime}=0.25 \mathrm{GeV}^{-2}$ where the single diffractive cross section dependence is: $\frac{d \sigma_{S D}}{d \xi} \propto \frac{1}{\xi^{\alpha(0)}}$, describes the single-sided sample well, giving confidence in the diffractive modelling. Thus, the measured $f_{d}=26.9_{-1.0}^{+2.5} \%$ is obtained using the default DL model.

The extrapolated cross section is therefore ${ }^{5}$ :

$$
\sigma_{\text {inel }}\left(\xi>\frac{m_{p}^{2}}{s}\right)=69.4 \pm 2.4(\exp ) \pm 6.9(\text { extr }) \mathrm{mb} .
$$

Fig.1(a) shows the proton-proton (and proton-antiproton) inelastic cross section as a function of $\sqrt{s}$ measured by different experiments at different centre of mass energy. No evidence of saturation at large $\sqrt{s}$ is observed yet. ${ }^{\mathrm{d}}$.

\footnotetext{
aThe MBTS counters span a pseudorapidty range $2.08<|\eta|<3.75$ symmetric on both sides of the ATLAS detector.

${ }^{\mathrm{b}}$ The threshold is set to $0.12 \mathrm{pC}$ while noise is well described by a gaussian centred at zero with a width of $0.02 \mathrm{pC}$.

${ }^{\mathrm{c}} \sigma_{S D}, \sigma_{D D}, \sigma_{C D}$ are respectively the single diffractive, the double diffractive and the central diffractive cross sections

${ }^{\mathrm{d}}$ In the interval between the conference and the writing of these proceedings, the ATLAS collaboration released the total cross section measurement from total elastic scattering using ALFA detectors $^{6}$. The measured value is in agreemnt with the values measured by TOTEM.
} 


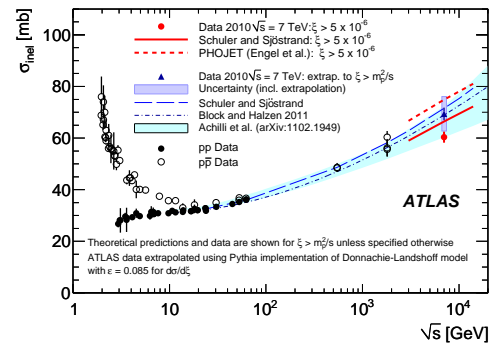

(a)

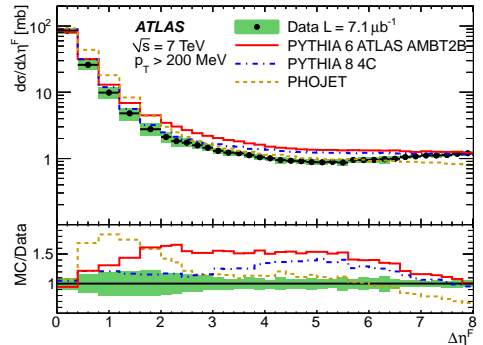

(b)

Fig. 1. (a) The measured inelastic $p p$ and $p \bar{p}$ cross section are shown for different centre-of-mass energies. The ATLAS measurement for $\xi>5 \times 10^{-6}$ is shown as the red filled circle. The extrapolation from the measured range to the full inelastic cross section is also shown (blue filled triangle $)^{5}$. (b) Inelastic cross section differential in forward gap size $\Delta \eta^{F}$ for charged particles with $P_{T}>200 \mathrm{MeV}$. The shaded bands represent the total uncertainties. The full lines show the predictions of PHOJET and the default versions of PYTHIA6 and PYTHIA8 ${ }^{7}$.

\section{Rapidity gap cross section}

Rapidity gaps are an evolution of the concept of single-sided events mentioned in the previous section. Rapidity gaps are identified by requiring no-particle activity in rings in $\eta$ of width 0.1 starting at the edge of ATLAS pseudorapidty acceptance $(\eta \pm 4.9)^{\mathrm{e}}$. By "no-particle activity" we mean that no deposit with energy above noise in the calorimeter and no charged track reconstructed with $P_{T}>200 \mathrm{MeV} / c$ is found ${ }^{7}$. The data sample is a subsample of the one used in the previous section. It corresponds to an integrated luminosity of $7.1 \pm 0.2 \mu \mathrm{b}^{-1}$. The mean number of interactions per bunch crossing is below 0.005. The measured differential cross section as a function of $\Delta \eta^{F}$ is unfolded back to hadron level and is compared with different MC predictions (Fig.1(b)). For small $\Delta \eta^{F}$ the non diffractive component is dominant and is exponentially suppressed. For $\Delta \eta^{F} \geq 3.5$ the differential cross section reaches a plateau with $d \sigma / d \eta^{F} \simeq 1 \mathrm{mb}$ per unit of pseudorapidity. It should be noted that none of the MC considered (PHOJET, PYTHIA ${ }^{8,9}$ describe well the measured differential cross section in the full $\Delta \eta^{F}$ range shown in Fig.1(b). The differential cross section can be expressed as a function of the Pomeron trajectory $\alpha_{\mathbb{P}}(t)=\alpha_{\mathbb{P}}(0)+\alpha_{\mathbb{P}}^{\prime} t$. Data lying in the $6<\Delta \eta^{F}<8$ range are used to estimate an effective Pomeron intercept $\left(\alpha_{\mathbb{P}}(0)\right)$ within the PYTHIA 8 MC. The DL intercept obtained after the fit for the $\mathbb{P}$ trajectory is:

$$
\alpha_{\mathbb{P}}(0)=1.058 \pm 0.003(\text { stat })_{-0.039}^{+0.034} \text { (syst) }
$$

The integrated inelastic cross section is obtained integrating the measured differential cross section over the $\Delta \eta^{F}$ distribution from zero to a maximum gap size

\footnotetext{
e Although some ATLAS subdetectors cover larger pseudorapidity, $|\eta|=4.9$ is the limit of acceptance for subdetectors that have an almost complete $2 \pi \phi$ coverage.
} 
$\Delta \eta_{\text {cut }}^{F}$

$$
\int_{0}^{\Delta \eta_{C u t}^{F}} \frac{d \sigma}{d \Delta \eta^{F}} d \Delta \eta^{F} \quad \text { with } 3<\Delta \eta_{C u t}^{F}<8 .
$$

which can be translated to the integrated inelastic cross section:

$$
\int_{\xi_{\text {cut }}}^{1} \frac{d \sigma}{d \xi_{X}} d \xi_{X}
$$

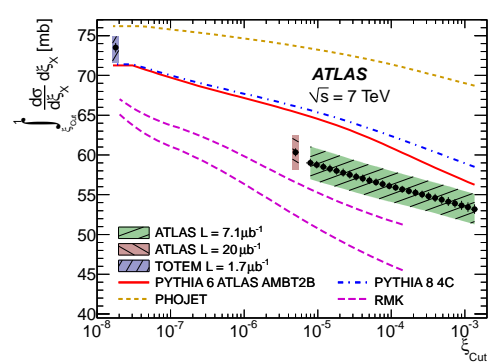

(a)

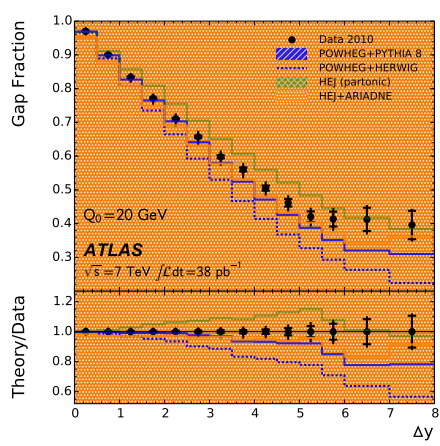

(b)

Fig. 2. (a) Inelastic cross section excluding diffractive processes with $\xi_{X}<\xi_{\text {Cut }}$, obtained by integration of the differential cross section from gap sizes of zero to a variable maximum. Measurements for $\xi_{\text {Cut }}$ single values from TOTEM and ATLAS are also reported ${ }^{7}$. (b) Measured gap ${ }^{10}$ fraction as a function of $\Delta Y$. The inner error bars represent statistical uncertainty while the outer error bars represent the quadrature sum of the systematic and statistical uncertainties. For comparison, the predictions from parton-level HEJ, HEJ+ARIADNE, POWHEG+PYTHIA and POWHEG+HERWIG are included.

The integrated inelastic cross section is shown as a function of $\xi_{\text {Cut }}$ in Fig.2(a), where it is also compared with the result reported in Sec.2 and with the TOTEM measurement of the full inelastic cross section ${ }^{11}$, derived from a measurement of the elastic cross section via the optical theorem.

The predictions of the default versions of the PYTHIA6, PYTHIA8 and PHOJET models are also shown, along with two versions of the RMK model ${ }^{12}$.

\section{Dijet production with central veto}

Measurements presented in the previous section are performed requiring no-particle activity in the rapidity gap. The perturbative QCD regime is investigated if, instead of the no-particle activity request, reconstructed jets are vetoed in the central region.

ATLAS has studied dijet production where no additional jet activity is present in the rapidity region between the two jets in $p p$ collisions at $\sqrt{s}=7 \mathrm{TeV}^{10,13}$. Jets are reconstructed using the anti-kt $\operatorname{algorithm}(R=0.6)$. The fraction of dijet events 
is defined as:

$$
f\left(Q_{0}\right)=\frac{\sigma_{j j}\left(Q_{0}\right)}{\sigma_{j j}},
$$

where $\sigma_{j j}$ is the inclusive cross section and $\sigma_{j j}\left(Q_{0}\right)$ is the dijet cross section where no additional jet with transverse momentum larger than $Q_{0}$ is present in the rapidity interval $\Delta Y$ bounded by the dijet system.

Gap fraction as a function of $\Delta Y$ is shown in Fig.2(b) for $Q_{0}=20 \mathrm{GeV}$. Experimental results are compared with state-of-the-art theoretical predictions: POWHEG and HEJ ${ }^{14}$ that provides a leading-log calculation of the perturbative terms that dominate the production of multijet events, when the jets span a large range in rapidity. Both generators are interfaced with simulations providing parton showering, hadronization and underlying event. None of the theoretical predictions agree well with the data across the full phase space considered; however, POWHEG+PYTHIA8 and HEJ+ARIADNE are found to provide the best agreement with the data. In the phase space regions studied here, large contributions from logarithmic functions of $\Delta Y$ and $\overline{P_{T}}$ must be summed to all orders to obtain accurate theoretical predictions. Two approaches are available: the Balitsky-FadinKuraev-Lipatov (BFKL) where the resummation is performed in terms of $\ln (1 / x)$, or the Dokshitzer-Gribov-Lipatov-Altarelli-Parisi (DGLAP) where terms of $\ln \left(Q^{2}\right)$ are summed up. This measurement is therefore sensible to which of the two approaches predict data with more accuracy. In addition, these measurements are of fundamental importance for understanding background processes for example, to the vector boson fusion (VBF) production of the Higgs boson. To discriminate the VBF production mode from the gluon fusion production process a central veto on dijets is applied in the event selection. An accurate model of the background greatly reduces systematics.

\section{Conclusions and Prospects}

Diffraction processes have been measured by the ATLAS experiment in $p p$ collisions during Run1 of LHC. In the upcoming Run2, the diffractive physics program will continue at $\sqrt{s}=13$; the largest centre-of-mass energy ever reached in $p p$ collisions. Subdetectors upgrades beyond Run2 will extend the ATLAS diffractive physics program. In particular, forward proton taggers with excellent timing resolution $(\mathcal{O}(10 \mathrm{ps}))$ will mitigate the impact of multiple interactions associating the correct primary interaction vertex to the elastically diffused proton. A larger dataset can thus be analyzed allowing the study of processes with smaller production cross sections.

\section{Acknowledgments}

It is a pleasure to thank the organizers of the conference for the stimulating scientific environment and for fruitful discussions. 


\section{References}

1. ATLAS Collaboration, The ATLAS Experiment at the CERN Large Hadron Collider, JINST 3, S08003 (2008).

2. A. Sidoti, Minimum Bias Trigger Scintillators in ATLAS, in INSTR 2014, (Novosibirsk, Russia, 2014).

3. R. Engel, Photoproduction within the two component dual parton model. 1. Amplitudes and cross-sections, Z. Phys. C 66, 203 (1995).

4. R. Engel and J. Ranft, Hadronic photon-photon interactions at high-energies, Phys. Rev. D 54, 4244 (1996).

5. ATLAS Collaboration, Measurement of the Inelastic Proton-Proton CrossSection at $\sqrt{s}=7 \mathrm{TeV}$ with the ATLAS Detector, Nature Commun. 2, 463 (2011).

6. ATLAS Collaboration, Measurement of the Total Cross Section from elastic scattering in pp collisions at $\sqrt{s}=7 \mathrm{TeV}$ with the ATLAS Detector, Submitted to Nucl. Phys. B (2014).

7. ATLAS Collaboration, Rapidity Gap Cross Sections measured with the ATLAS Detector in pp Collisions at $\sqrt{s}=7 \mathrm{TeV}$, Eur. Phys. J. C72, 1926 (2012).

8. S. M. T. Sjöstrand and P. Skands, PYTHIA 6.4 Physics and Manual, JHEP 0605, 026 (2006).

9. S. M. T. Sjöstrand and P. Skands, A Brief Introduction to PYTHIA 8.1, Comput.Phys.Commun. 178, 852 (2008).

10. ATLAS Collaboration, Measurement of the production and azimuthal decorrelation of dijets with a jet veto in pp collisions at $\sqrt{s}=7 \mathrm{TeV}$ using the ATLAS detector (2014).

11. TOTEM Collaboration, Measurement of proton-proton elastic scattering and total cross-section at $\sqrt{s}=7 \mathrm{TeV}$, Europhys. Lett. 96, 21002 (2011).

12. M. Ryskin, A. Martin and V. Khoze, High-energy strong interactions: from 'hard' to 'soft', Eur. Phys. J. C71, 1617 (2011).

13. ATLAS Collaboration, Measurement of dijet production with a veto on additional central jet activity in pp collisions at $\sqrt{s}=7 \mathrm{TeV}$ using the ATLAS detector, JHEP 1109, 053 (2011).

14. J. R. Andersen and J. M. Smillie, Multiple Jets at the LHC with High Energy Jets, 1106, 010 (2011). 